\title{
Identification of Potential Hub Genes and Therapeutic Drugs in Malignant Pleural Mesothelioma by Integrated Bioinformatics Analysis
}

\author{
Xiangxin Zhang ${ }^{a} \quad$ Liu Yang $^{\text {a }}$ Wei Chen ${ }^{b}$ Ming Kong ${ }^{c}$ \\ ${ }^{a}$ School of Medicine, Shihezi University, Shihezi, China; ${ }^{b}$ Department of Anaesthetic Operating Room, Provincial \\ Otolaryngology Hospital Affiliated to Shandong University, Shandong Provincial Western Hospital, Jinan, China; \\ 'Department of Thoracic Surgery, Provincial Otolaryngology Hospital Affiliated to Shandong University, Shandong \\ Provincial Western Hospital, Jinan, China
}

\section{Keywords}

Bioinformatics $\cdot$ GEO database $\cdot$ Malignant pleural mesothelioma $\cdot$ Hub genes · Drugs

\begin{abstract}
Introduction: Malignant pleural mesothelioma (MPM) is closely linked to asbestos exposure and is an extremely aggressive tumor with poor prognosis. Objective: Our study aimed to elucidate hub genes and potential drugs in MPM by integrated bioinformatics analysis. Methods: GSE42977 was download from the Gene Expression Omnibus (GEO) database; the differentially expressed genes (DEGs) with adj.p value $<0.05$ and $|\log \mathrm{FC}| \geq 2$ were identified. Gene Ontology (GO) and Kyoto Encyclopedia of Genes and Genomes (KEGG) pathway analyses were performed by DAVID database. The STRING database was used to construct a protein-protein interaction network, and modules analysis and hub genes acquisition were performed by Cytoscape. The Gene Expression Profiling Interactive Analysis (GEPIA) database was used to assess the impact of hub genes on the prognosis of MPM patients. The Drug-Gene Interaction database (DGIdb) was used to select the related drugs. Results: A total of 169 upregulated and 70 downregulated DEGs were identified. These DEGs are enriched in the pathway of extracellular matrix-receptor interaction, focal adhesion, PI3K-Akt signaling pathway, and PPAR signaling pathway. Finally, 10 hub genes (CDC20, CDK1, UBE2C, TOP2A, CCNB2, NUSAP1, KIF20A,
\end{abstract}

AURKA, CEP55, and ASPM) were identified, which are considered to be closely related to the poor prognosis of MPM. In addition, 119 related drugs that may have a therapeutic effect on MPM were filtered out. Conclusion: These discovered genes and small-molecule drugs provide some new ideas for further research on MPM.

(c) 2020 The Author(s) Published by S. Karger AG, Basel

\section{Introduction}

Malignant pleural mesothelioma (MPM) is a rare, incurable, and aggressive cancer of the pleural surface with a median survival time of $<2$ years [1]. Its main cause is related to asbestos exposure; it is not easy to find in an early stage. Its treatment includes surgery, chemotherapy, and radiotherapy. Moreover, the large majority of patients do not have the opportunity to undergo surgery due to the advanced stage of the disease and related complications. Thus, identifying molecular biomarkers is critically important for early diagnosis, prevention, and personalized therapy.

Recently, with the rapid development of microarray technology, bioinformatics analysis has been extensively applied to explore mechanisms of a variety of disorders, particularly in neoplasms. Integrating and reanalyzing these genomic data offer possibilities for identifying certain disease-related biomarkers. Recently, some research-

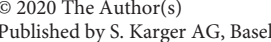

This is an Open Access article licensed under the Creative Commons Attribution-NonCommercial-4.0 International License (CC BY-NC) (http://www.karger.com/Services/OpenAccessLicense), applicable to the online version of the article only. Usage and distribution for commercial purposes requires written permission. 
Table 1. The 239 DEGs were identified from GSE42977, including 169 upregulated genes and 70 downregulated genes

\begin{tabular}{ll}
\hline DEGs & Gene name \\
\hline Upregulated & MYOC, CLC, FCGR3B, LPL, CA4, CXCR2, ADIPOQ, THRSP, AQP7P1, AQP7P2, DMRT2, LIPE, PROK2, \\
& FXYD1, GPD1, TF, VNN3, CMTM2, TUSC5, CD36, HMFN0839, S100P, C18orf34, GPAM, AQP7P2, TIMP4, \\
& NTRK2, ATP1A2, FAM13A1, SERPINA5, FLJ22655, ERAF, SOX17, MYOM1, LOC400258, LOC644322, CIDEA, \\
& IL8RA, HBD, PLIN, RBP7, CIDEC, CASQ2, SORBS1, SLC7A10, PADI4, ALDH1L1, G0S2, IL20, KIAA1881, \\
& RDH5, PCOLCE2, S100A9, ECRG4, C6, PPARG, HBA1, LEP, CDO1, VSIG2, DEFA1, CCDC3, DEFA3, \\
& FAM107A, APOLD1, SLC19A3, LMO3, DPT, HSPB6, CAMP, S100A8, CKMT2, SELE, PDK4, SLC25A25, HBG1, \\
& FLJ25530, FGR, ACACB, MAOA, HBG2, MOSC1, LGALS12, HBB, RBP4, LOC648517, ALOX15, TNNT3, \\
& NR4A1, CXCL2, RSPO3, PI16, NLF2, NR4A3, CFD, SRL, TNMD, FABP4, ECHDC3, CX3CL1, GPX3, PTGER3, \\
& APOD, CCL14, TGFBR3, SCARA5, MMP25, FCN1, CLDN5, VNN2, TSPAN8, IL6, ABCA8, COX7A1, RASD1, \\
& MYOT, ADH1B, IL8, AQP9, AXUD1, CTSG, CMYA5, CEBPA, ADAMTS1, GYG2, MYH11, FMO2, MYH7, \\
& DARC, TNNC2, ADAMTS4, CA3, RGS2, MRAP, TCAP, MGST1, FHL1, FOSB, WISP2, NR4A2, MYOZ1, SYT4, \\
& MYL2, AKR1C3, MB, MYBPC1, NEB, SPARCL1, ALDH1A1, MYLPF, XM_373497.4, HP, SFRP1, MYH2, \\
& KBTBD10, ADH1A, NELL2, CKM, C10orf116, PLAC9, MT1M, MYL1, MYL1, CPB1, SLN, CCL19, TNNC1, \\
& VIPR2, PRG4 \\
& FSCN1, COL1A1, TRPV4, P4HA1, COL5A1TOP2A, CCNB2, NUSAP1, IQGAP3, UHRF1, ASPM, TUBB3, PRC1, \\
& TYMS, KIAA0101, CDC20, MELK, KIF20A, GAL3ST4, CDC2, UNG2, COL3A1, TFAP2A, UBE2C, C20orf129, \\
& MDK, CDH11, PLOD2, STK6, TRIP13, CENPF, CTHRC1, TK1, CEP55, FZD2, CDCA5, SLC12A8, COL11A1, \\
& PTTG1, SCG2, PNMA2, HAMP, IRX3, C7orf10, LOC644743, COL5A2, LOC647954, FAP, TBC1D2, CDH2, \\
& SERPINE1, HIST1H2BD, MMP11, RIS1, GJB2, KIAA1199, BCAT1, FBN2, UCHL1, COL10A1, FNDC1, FCGBP, \\
& LOC144501, SPP1, COMP, POSTN, CPA4, LAMC2, TNFSF7, NMU \\
\hline Downregulated & \\
\hline
\end{tabular}

ers have been using bioinformatics methods to analyze the microarray of MPM [2, 3]. Many bioinformatical studies, for example on ovarian cancer, have been proven to be effective and reliable $[4,5]$, which means integrated bioinformatics analysis may assist in exploring the biomarkers and potential mechanisms of MPM.

In this study, GSE42977 were downloaded from the Gene Expression Omnibus (GEO) database for the identification of differentially expressed genes (DEGs) between MPM tissues and normal pleural tissues. Gene Ontology (GO) functional annotation analysis and Kyoto Encyclopedia of Genes and Genomes (KEGG) pathway enrichment analysis were used for the screened DEGs. Then, we established a protein-protein interaction (PPI) network and used Cytoscape for selected hub genes related to MPM. We validated the hub genes with the Gene Expression Profiling Interactive Analysis (GEPIA) online database. Finally, through the Drug-Gene Interaction database (DGIdb), we identified 119 related drugs that may have a therapeutic effect on MPM. In conclusion, these results provide novel and reliable biomarkers for screening, diagnosis, and prognosis, as well as potential therapeutic targets for MPM.

\section{Methods}

Microarray Data Information and DEG Identification

The gene expression profiles GSE42977 of mesothelioma in the GEO database (http://www.ncbi.nlm.nlh.gov/geo/) [6] were used, based on GPL6790 (Illumina HumanRef-6 version 2.0 expression bead chip). The GSE42977 was submitted by De Rienzo et al. [7] in 2013, including 24 MPM epithelioid tissues and 7 normal pleural tissues. GEO2R is an interactive web tool that allows users to compare 2 or more groups of samples in a GEO series to identify genes that are differentially expressed across experimental conditions. Results are presented as a table of genes ordered by significance [6]. In this study, GEO2R was used to identify DEGs between MPM and normal pleura. The selection criteria for DEGs are: adj. $p$ $<0.05$ and $|\log \mathrm{FC}| \geq 2$.

\section{GO and Pathway Enrichment Analysis}

The DAVID online database (http://david-d.ncifcrf.gov/) [8] was used to perform GO and KEGG pathway analyses. GO term enrichment analysis includes biological process, cellular component (CC), and molecular function (MF). We put the list of upregulated DEGs and downregulated DEGs into the search box. After selecting the official gene symbol and species background (Homo sapiens), click submit to obtain the GO and KEGG pathway analysis results of these genes.

\section{Construction of the PPI Network}

The STRING database was used to provide a key assessment of protein-protein functional associations [9]. To further explore the relationships between the selected genes at the protein level, the STRING (version 11, http://string-db.org/) online database was used. In this study, the confidence score was set to the highest (score 0.900). We downloaded the integrated interaction from STRING. Cytoscape [10] is a software tool for the visual exploration of biomedical networks composed of proteins, genes, and other types of interactions, supported by diverse annotations and experimental data. We used the Cytoscape software version 3.6.1 to visualize and analyze the interaction network.

Identification of the Hub Genes

To select the hub genes, the cytoHubba [11] of the Cytoscape software was used, which can predict and explore important nodes 
and subnetworks in a given network using several topological algorithms. Among 12 computing methods, Maximal Clique Centrality was selected to explore hub genes.

Hub Genes Validation by GEPIA

GEPIA (https//:gepia.cancer-pku.cn) [12] is a newly developed interactive web server for analyzing the RNA sequencing expression data of 9,736 tumors and 8,587 normal samples from the TCGA and the GTEx projects. In addition, GEPIA provides customizable functions, such as tumor/normal differential expression analysis, profiling according to cancer types or pathological stages, patient survival analysis, similar gene detection, correlation analysis, and dimensionality reduction analysis. Since there is no separate MPM dataset in the GEPIA database and MPM belongs to a special type of mesothelioma (MESO), we chose MESO, a large dataset, to initially explore the possible impact of these hub genes on the prognosis of MPM patients. We calculated the hazard ratio based on Cox PH Model and drew the 95\% CI.

\section{Drug-Gene Interaction Analysis}

The online DGIdb (http://www.dgidb.org/) now contains $>40,000$ genes and $>10,000$ drugs, involving $>15,000$ drug-gene interactions [13]. We put the obtained hub genes together in the search box to retrieve all known or potential small-molecule substances related to these genes.

\section{Results}

\section{Identification of DEGs}

Through the GEO2R analysis tool that comes with the GEO database and the set DEG selection criteria adj. $p<$ 0.05 and $|\log \mathrm{FC}| \geq 2$, we finally identified a total of 239 DEGs from the GSE42977 gene expression profile, including 169 upregulated genes and 70 downregulated genes (Table 1). The visualization of these DEGs is shown by the volcano map (Fig. 1).

\section{Enrichment Analysis}

DAVID online gene annotation results show that the upregulated DEGs (Fig. 2a) are mainly related to biological processes, such as muscle filament sliding, oxygen delivery, muscle contraction, myocardial contraction, and cell-to-necrosis factor; CC are related to the extracellular space and extracellular region, hemoglobin complex, muscle myosin complex, and extracellular vesicles; in addition, MF shows that the structural components of muscle, oxygen transporter activity, oxygen binding, heparin binding, and arachidonic acid binding are related to the upregulated differential genes. The biological processes of downregulated DEGs (Fig. 2b) include extracellular matrix (ECM) tissue, collagen fibril tissue, collagen breakdown process, skeletal system development, and mitotic mitosis; $\mathrm{CC}$ is mainly associated with collagen trimer, proteinaceous ECM, ECM, endoplasmic reticulum lumen, and the extracellular region; MF is related to ECM structural constituent, plateletderived growth factor binding, heparin binding, drug

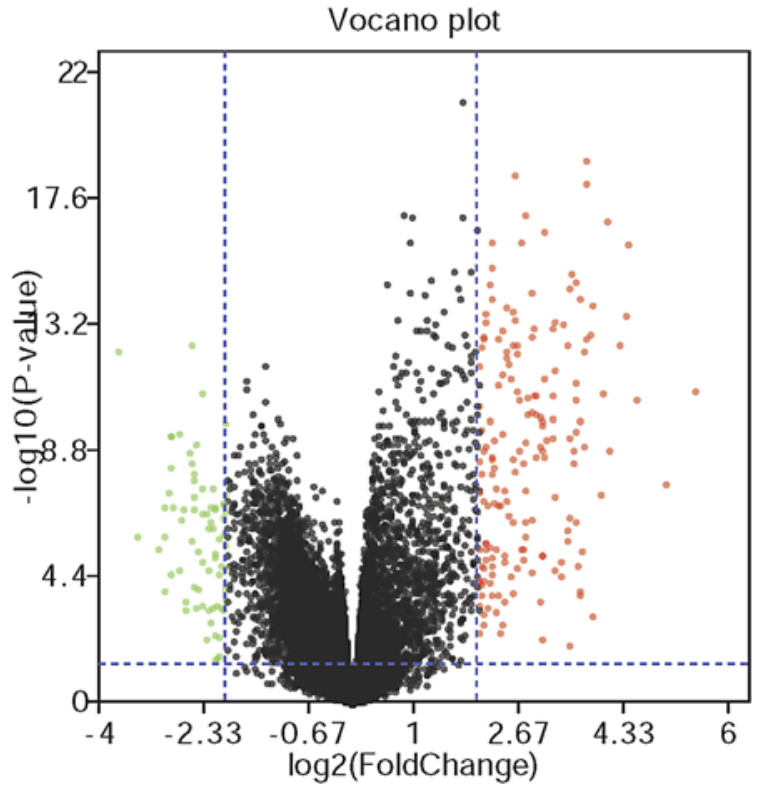

Fig. 1. Volcano plot of GSE42977. Cutoff criteria: adj. $p$ value $<0.05$ and $\mid \log \mathrm{FCl} \geq 2$. The upregulated genes are red, and the downregulated genes are green, while not significantly changed genes are grey.

binding, and microtubule binding. In addition, pathway analysis showed that these genes were highly related to ECM-receptor interaction, focal adhesion, protein digestion and absorption, amoebiasis, PI3K-Akt signaling pathway, and PPAR signaling pathway (Fig. 2c, d; Table 2).

\section{PPI Network Construction and Module Analysis}

To establish the interactions of these DEGs in MPM, we used the STRING online database to construct a PPI network. A total of 111 nodes and 296 edges were involved in the PPI network (Fig. 3a). On the basis of MCODE, we identified 5 modules in the network with the sitting Kcore $>5$ (Fig. 3b-f; Table 3). Module 1 included 14 nodes and 86 edges. The DEGs of module 1 were significantly enriched in oocyte meiosis, cell cycle, and p53 signaling pathway. Module 2 consisted of 8 nodes and 28 edges. The DEGs of module 2 were enriched in chemokine signaling pathway, cytokine-cytokine receptor interaction, and NOD-like receptor signaling pathway. Module 3 also consisted of 8 nodes and 28 edges. The DEGs of module 3 were enriched in cardiac muscle contraction, hypertrophic cardiomyopathy, and dilated cardiomyopathy. Module 4 included 9 nodes and 32 edges. The DEGs of module 4 were enriched in protein digestion and absorption, ECM-receptor interaction, and PI3K-Akt signaling path- 



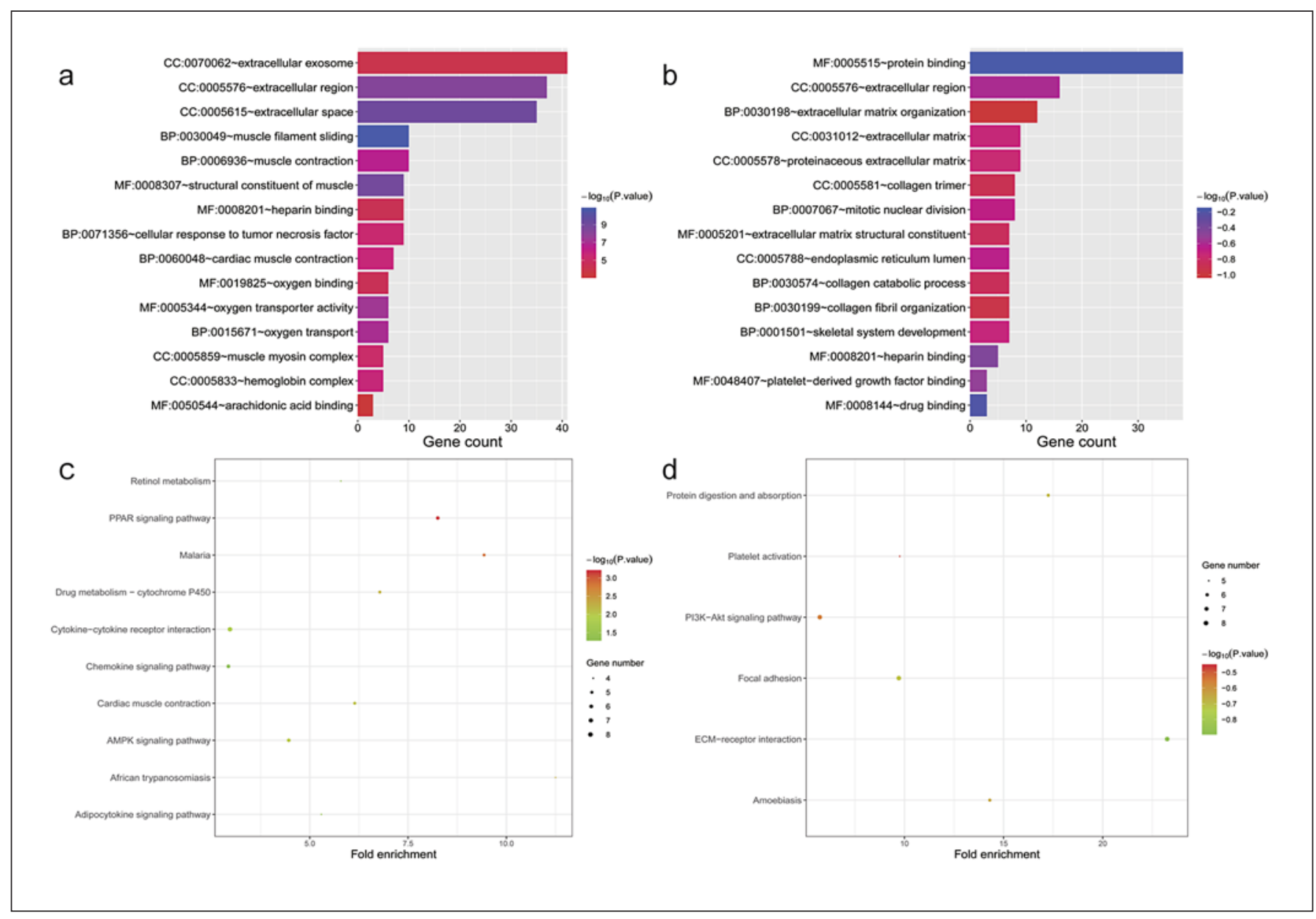

Fig. 2. Functional analysis of DEGs in MPM. a Top 5 GO analysis of upregulated genes. $\mathbf{b}$ Top 5 GO analysis of downregulated genes. $\mathbf{c}$ KEGG pathway analysis of upregulated genes. $\mathbf{d}$ KEGG pathway analysis of downregulated genes.

way. Module 5 consisted of 6 nodes and 15 edges. The DEGs of module 5 were enriched in the HIF-1 signaling pathway and the Toll-like receptor signaling pathway.

\section{Hub Genes Identification}

The cytoHubba of the Cytoscape software was used to select the significant hub genes among the obtained DEGs. The Maximal Clique Centrality method was used to select the top 10 genes of the PPI network. Then, we used a connectivity degree in the PPI network to evaluate the top 10 genes we just selected. The results showed that cell division cycle 20 (CDC20) and cyclin-dependent kinase 1 (CDK1) are the most outstanding genes with connectivity degree $=19$, followed by ubiquitin-conjugating enzyme E2 C (UBE2C, degree = 18), topoisomerase II alpha $($ TOP2A, degree $=16)$, cyclin B2 $(\mathrm{CCNB} 2$, degree = 15), nucleolar and spindle associated protein 1 (NUSAP1, degree $=14)$, kinesin family member $20 \mathrm{~A}(\mathrm{KIF} 20 \mathrm{~A}$, degree $=14)$, aurora kinase A (ARUKA, degree $=14)$, centrosomal protein $55($ CEP55, degree $=13)$, and assembly factor for spindle microtubules (ASPM, degree $=13$ ). It is worth noting that AURKA and CDK1 are the official symbols of STK6 and CDDC2, which are all downregulated DEGs.

\section{Survival Analysis of Hub Genes}

In order to further understand the possible impact of these hub genes on the survival of MPM patients, we verified these genes in a large dataset of MESO (Fig. 4). The results show that for ASPM (HR 1.9, $p=0.011$ ), AURKA (HR 2.8, $p=3.2 \mathrm{e}-05)$, CCNB2 (HR 4.1, $p=3.1 \mathrm{e}-08$ ), CDC20 (HR 3.4, $p=1.2 \mathrm{e}-06$ ), CDK1 (HR 3.5, $p=7.6 \mathrm{e}-$ 07), CEP55 (HR 4.1, $p=1.7 \mathrm{e}-08$ ), KIF20A (HR 3.8, $p=$ 6.3e-07), NUSAP1 (HR 2.7, $p=7 \mathrm{e}-05$ ), TOP2A (HR 5.3, $p=2.9 \mathrm{e}-10$ ), and UBE2C (HR 3.1, $p=-6.4 \mathrm{e}-06$ ), the survival rate of these high-expression groups was significantly lower than for the low-expression groups. In addition, the analysis of Cox regression factors further showed that high expression of these genes is a prognostic risk factor relative to low expression. 
Table 2. Results of pathway analysis of DEGs

\begin{tabular}{|c|c|c|c|c|}
\hline Category & Term & Count & $p$ value & Genes \\
\hline \multirow{7}{*}{$\begin{array}{l}\text { Upregulated } \\
\text { genes KEGG } \\
\text { pathway }\end{array}$} & hsa03320: PPAR signaling pathway & 6 & $6.79 \mathrm{E}-04$ & $\begin{array}{l}\text { LPL, CD36, SORBS1, PPARG, FABP4, } \\
\text { ADIPOQ }\end{array}$ \\
\hline & hsa05144: malaria & 5 & $1.72 \mathrm{E}-03$ & IL6, CD36, HBA1, HBB, SELE \\
\hline & hsa05143: African trypanosomiasis & 4 & 4.98E-03 & IL6, HBA1, HBB, SELE \\
\hline & hsa00982: drug metabolism - cytochrome P450 & 5 & $5.70 \mathrm{E}-03$ & FMO2, MAOA, ADH1B, ADH1A, MGST1 \\
\hline & hsa04260: cardiac muscle contraction & 5 & $8.04 \mathrm{E}-03$ & MYL2, COX7A1, TNNC1, MYH7, ATP1A2 \\
\hline & hsa04152: AMPK signaling pathway & 6 & $9.61 \mathrm{E}-03$ & $L E P, C D 36, P P A R G, A C A C B, L I P E$ \\
\hline & hsa04060: cytokine-cytokine receptor interaction & 8 & $1.41 \mathrm{E}-02$ & $\begin{array}{l}\text { LEP, IL6, CCL14, CXCL2, CCL19, CXCR2, } \\
\text { CX3CL1, IL20 }\end{array}$ \\
\hline \multirow{6}{*}{$\begin{array}{l}\text { Downregulated } \\
\text { genes KEGG } \\
\text { pathway }\end{array}$} & hsa04512: ECM-receptor interaction & 8 & $2.19 \mathrm{E}-08$ & $\begin{array}{l}\text { COMP, COL3A1, LAMC2, COL1A1, } \\
\text { COL11A1, COL5A2, COL5A1, SPP1 }\end{array}$ \\
\hline & hsa04510: focal adhesion & 8 & 7.90E-06 & $\begin{array}{l}\text { COMP, COL3A1, LAMC2, COL1A1, } \\
\text { COL11A1, COL5A2, COL5A1, SPP1 }\end{array}$ \\
\hline & hsa04974: protein digestion and absorption & 6 & $1.63 \mathrm{E}-05$ & $\begin{array}{l}\text { COL3A1, COL1A1, COL11A1, COL5A2, } \\
\text { COL5A1, COL10A1 }\end{array}$ \\
\hline & hsa05146: amoebiasis & 6 & 4.02E-05 & $\begin{array}{l}\text { COL3A1, LAMC2, COL1A1, COL11A1, } \\
\text { COL5A2, COL5A1 }\end{array}$ \\
\hline & hsa04151: PI3K-Akt signaling pathway & 8 & $2.16 \mathrm{E}-04$ & $\begin{array}{l}\text { COMP, COL3A1, LAMC2, COL1A1, } \\
\text { COL11A1, COL5A2, COL5A1, SPP1 }\end{array}$ \\
\hline & hsa04611: platelet activation & 5 & $1.32 \mathrm{E}-03$ & $\begin{array}{l}\text { COL } 3 A 1, \text { COL1A1, COL11A1, COL5A2, } \\
\text { COL5A1 }\end{array}$ \\
\hline
\end{tabular}

Table 3. The top 5 significant modules

\begin{tabular}{lllll}
\hline Modules & Nodes & Edges & Score & Genes \\
\hline Module 1 & 14 & 86 & 13.231 & $\begin{array}{l}\text { CDC20, TOP2A, CDK1, CENPF, UBE2C, CEP55, MELK, NUSAP1, ASPM, } \\
\text { KIF20A, PRC1, CCNB2, AURKA, PTTG1 }\end{array}$ \\
\hline Module 2 & 8 & 28 & 8.000 & NMU, CXCR1, PTGER3, CXCL2, CX3CL1, CXCR2, CXCL8, CCL19 \\
\hline Module 3 & 8 & 28 & 8.000 & MYL2, TNNC1, TNNC2, TNNT3, NEB, MYL1, MYBPC1, TCAP \\
\hline Module 4 & 9 & 32 & 8.000 & $\begin{array}{l}\text { P4HA1, COL11A1, COL10A1, PLOD2, COL5A2, COL5A1, COL1A1, } \\
\text { PCOLCE2, COL3A1 }\end{array}$ \\
\hline Module 5 & 6 & 15 & 6.000 & SPARCL1, SCG2, CDH2, TF, SPP1, IL6 \\
\hline
\end{tabular}

\section{Drug-Gene Interaction Analysis}

Through the DGIdb, we selected a total of 119 drugs related to 3 genes (online suppl. Table 1, see www. karger.com/doi/10.1159/000510534). These drugs mainly interact with the 3 genes CDK1, TOP2A, and AURKA. We selected 31 drugs with higher scores based on the comprehensive score on the website and finally used the STITCH [14] website for visual processing (Fig. 5).

\section{Discussion}

MPM is closely linked to asbestos exposure and is an extremely aggressive tumor with poor prognosis. Thus, identifying molecular biomarkers is critically important for early diagnosis, prevention, and personalized therapy. In this study, we analyzed the expression of genes in GSE42977 microarray datasets based on MPM epithelioid tissue and normal pleural tissue controls. A total of 


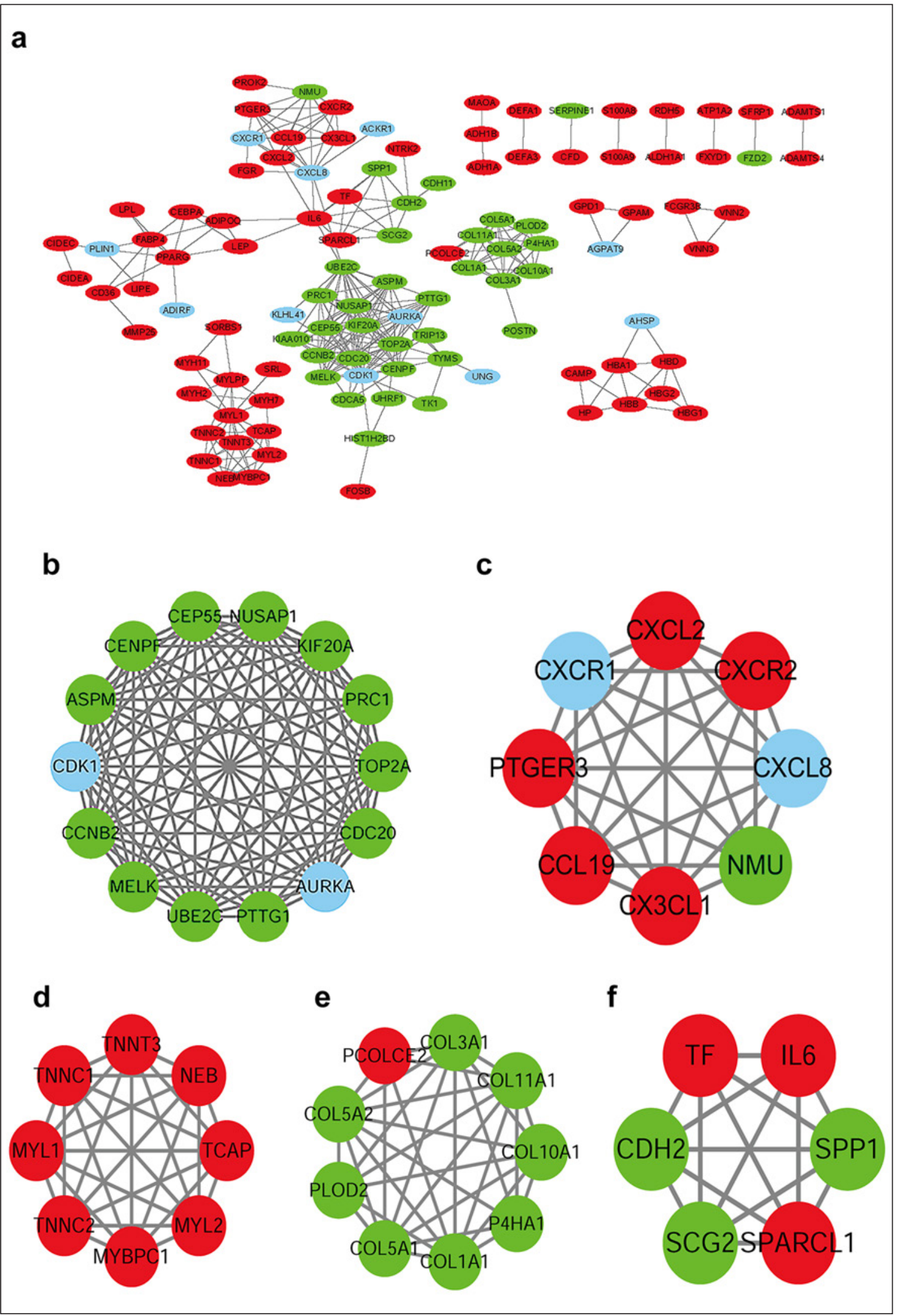

Fig. 3. DEGs PPI network complex in MPM. a All DEGs interaction networks. b-f The most significant modules selected from the PPI network. Red node indicates the upregulated gene and green node indicates the downregulated gene. The blue nodes PLIN1,
ADIRF, CXCR1, CXCL8, ACKR1, AGPAT9, AHSP, KLHL41, UNG, AURKA, and CDK1 are respectively the official symbol of PLIN, C10ORF116, IL8RA, IL8, DARC, HMFN0839, ERAF, KBTBD10, UNG2, STK6, and CDC2. 



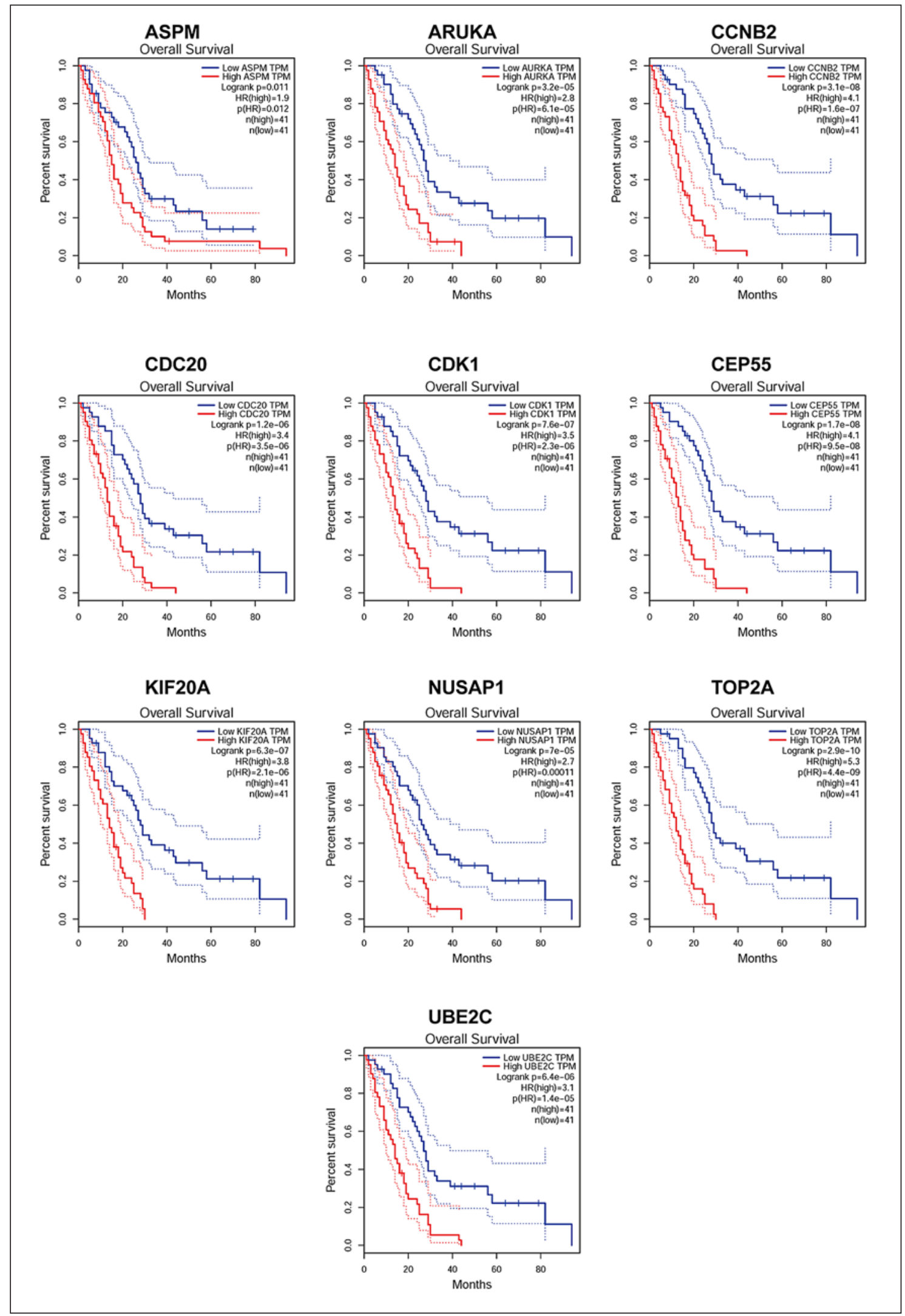

Fig. 4. Overall survival analyses of hub genes of MPM patients in GEPIA. $p<0.05$ was considered statistically significant. 


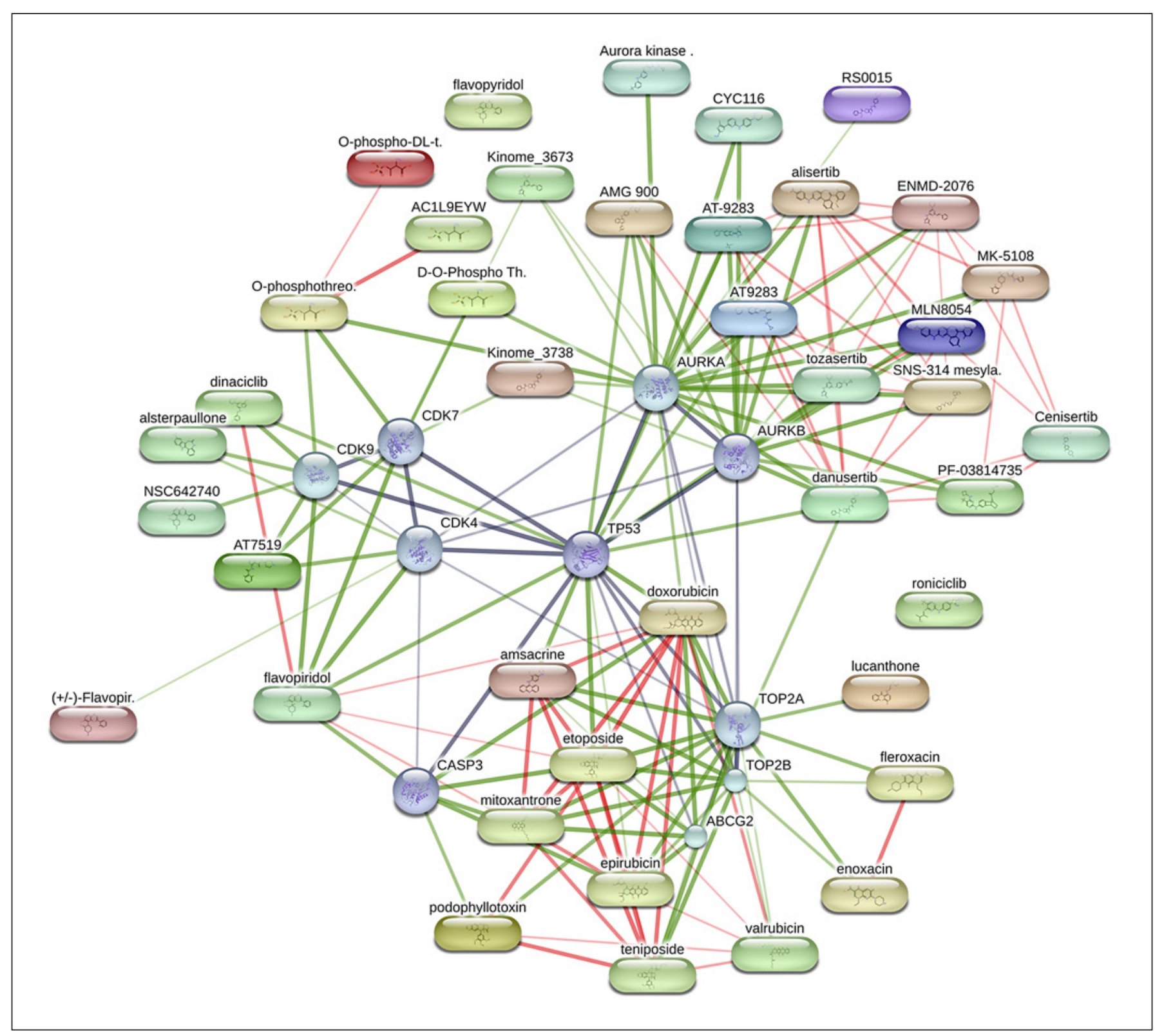

Fig. 5. Drug-gene interaction network. Stronger associations are represented by thicker lines. Protein-protein interactions are shown in grey, chemical-protein interactions in green, and interactions between chemicals in red.

239 common DEGs were identified (169 upregulated and 70 downregulated), with adj.p value $<0.05$ and $|\log \mathrm{FC}|$ $\geq 2$. Subsequently. We utilized bioinformatics methods to deeply explore these DEGs, including GO term enrichment, signaling pathway enrichment, PPI network construction, hub genes selection, and survival analysis. Finally, 10 hub genes were selected (TOP2A, CDC20, CDK1, UBE2C, CCNB2, NUSAP1, KIF20A, AURKA, CEP55, and ASPM). TOP2A gene encodes a DNA topoisomerase, an enzyme that controls and alters the topologic states of DNA during transcription. According to survival analysis, high expression of TOP2A is associated with a poor prognosis of MPM. According to some stud- ies, high expression levels of TOP2A exhibited prognostic importance in various carcinomas, such as prostate cancer [15] and breast cancer [16]. CDC20 appears to act as a regulatory protein interacting with several other proteins at multiple points in the cell cycle. It has been identified as a key candidate gene in a variety of cancers based on many bioinformatics studies [17, 18]. High expression levels of CDC20 have been reported to be related to poor prognosis for multiple tumors $[19,20]$, which is consistent with the results of this article's survival analysis. $\mathrm{CDK} 1$ is also known as CDC2; CDC28A. The protein encoded by this gene is a member of the Ser/Thr protein kinase family. It is essential for G1/S and G2/M phase 
transitions of the eukaryotic cell cycle. In many studies, high expression of CDK1 has been considered to be associated with poor prognosis of tumors [19], which is consistent with the results of this study. UBE2C is considered to play an important role in the tumorigenesis of many cancers and to promote cell cycle progression. Overexpression of UBE2C has been reported in many tumors, such as esophageal squamous cell carcinoma [21], ovarian carcinoma [22], and rectal carcinoma [23]. According to our results, overexpression of UBE2C was significantly correlated with overall survival of MPM patients. Many tumors are closely related to cyclins. CCNB2 is a member of the cyclin family, specifically the B-type cyclins. The B-type cyclins, B1 and B2, associate with p34cdc2 and are essential components of the cell cycle regulatory machinery. In our study, we also found CCNB2 was enriched in the pathway of oocyte meiosis, cell cycle, and p53 signaling pathway. Many researchers have reported that a high expression level of CCNB2 is associated with multiple tumors and poor prognoses, such as in hepatocellular carcinoma [24] and bladder cancer [25]. In our study, we also found that a high expression level of CCNB2 is related to poor prognosis of MPM. NUSAP1 is a nucleolar-spindle-associated protein and plays a role in spindle microtubule organization. Research by Liu et al. [26] has shown that a high expression level of NUSAP1 predicts poor prognosis in colon cancer. Our results also showed that a high expression of NUSAP1 leads to poor prognosis. KIF20A is well known as one of the key proteins in mitosis. Many recent studies have shown that KIF20A is regarded as a key gene in many tumors based on the bioinformatics analysis, such as hepatocellular carcinoma [27] and ovarian cancer [28]. AURKA is a protein-coding gene that plays an important role in regulating many of the processes that are pivotal to mitotic progression and is reported as a hub gene of many cancers, such as breast cancer, colorectal carcinoma, gastric cancer, and liver cancers [29-32]. Our studies showed that overexpression of AURKA leads to poor prognosis, which is consistent with Schneider et al.'s [33] study which proved that the expression of the mitosis-associated gene AURKA is associated with the prognosis of non-small cell lung cancer patients. Therefore, we have reason to speculate that AURKA is likely to be a potential biomarker for MPM. CEP55 is a key regulator of cytokinesis, and its high expression level is linked to genomic instability; it is regarded as a potential biomarker of various cancers. Recently, many studies have suggested that CEP55 was associated with multiple cancers. For example, Lin et al. [34], based on bioinformatics analysis, showed that CEP55 may play an important role in the oncogenesis and progression of HER-2-positive breast cancer. Liu et al.'s [35] research showed that CEP55 is closely related to gastric cancer and may become a potential therapeutic target or biomarker. In this study, we found that CEP55A is closely related to the survival time of MPM. We think it may play an important role in the process of MPM. ASPM is essential for normal mitotic spindle function in embryonic neuroblasts. Overexpression of ASPM is a molecular marker predicting the enhanced metastatic potential of hepatocellular carcinoma with poor prognosis [36]. In prostate cancer, high ASPM expression has also been reported to be correlated with tumor progression and to predict poor outcomes [37]. Our research also suggests that ASPM is associated with poor prognosis for MPM.

We verified the selected key genes on GEPIA, and the results showed that these key genes are all related to the poor prognosis of MPM. Through the DGIdb online database, a total of 119 drugs with potential therapeutic effects on MPM were selected. All data resources are from the NCI, DrugBank, and other drug-related databases. The main relationship between these drugs and genes is inhibitors, and some of them are binder and agonist. These related drugs are mainly related to CDK1, AURKA, and TOP2A.

Roniciclib is a type 1 pan-cyclin-dependent kinase $(\mathrm{CDK})$ inhibitor with potential efficacy in various cancer models [38]. Related research proves that roniciclib can be used as a potential drug for the treatment of patients with highly differentiated thyroid cancer [39]. Alvocidib is a CDK1 inhibitor, and related reports have shown that the drug has a strong therapeutic effect on acute myelocytic leukemia in clinical trials [40]. Alvocidib has encouraging clinical activity in inhibiting newly diagnosed and relapsed refractory acute myelocytic leukemia [41]. Dinaciclib has a certain effect in the treatment of pulmonary hypertension [42]. Currently, the CDK inhibitors alvocidib and dinaciclib are still in the clinical trial stage for various leukemias and solid tumors [40, 43, 44]. Alsterpaullone is a small-molecule CDK inhibitor that can regulate cell cycle progression [45] and may have a positive effect on reversing paclitaxel-induced drug resistance of ovarian cancer cells [46]. AT-7519, as part of adjuvant treatment of cervical cancer, plays a role in the control of cervical cancer [47]. Based on an animal experiment, AT-7519 has a significant tumor regression effect on MYCN-dependent neuroblastoma [48]. As for AURKA, MK-5108 has effective antiproliferative activity in lung cancer cell lines used alone and in combination with chemotherapy [49]. The combined use of alisertib and paclitaxel has been shown to have a positive effect on relapsed or refractory small cell lung cancer [50]. In addition, alisertib is also regarded as a new option for the treatment of acute myeloid leukemia [51]. In the research on breast cancer [52] and ovarian cancer [53], some progress has been made at the cellular level and in animal experiments, but there is still a lack of reliable clinical evidence. After the failure of previous systemic chemotherapy, single-use danusertib shows antitumor activity in com- 

mon solid tumors, but the effect is more limited [54]. ENMD-2076 is a novel, orally active molecule. Clinical trials related to breast cancer [55] and ovarian cancer [56] have been carried out, but more experimental data are still needed to support the efficacy of the drug. AMG-900 was only proved to have a certain effect on adrenocortical tumors at the cellular level, but there still is a lack of clinical evidence to support this [57]. SNS-314 has proved to have strong anti-tumor activity in mouse experiments [58]. Teniposide is a semisynthetic derivative of podophyllotoxin with a broad spectrum of in vivo antitumor activity. Amsacrine-related research is mainly for the treatment of acute myelocytic leukemia. The application of valrubicin has been reported to have benefits for the treatment of skin tumors [59], and it also has a certain effect on some bladder cancers [60]. Epirubicin has a certain effect on the combined treatment of breast cancer, and there is certain evidence in many clinical trials $[61,62]$. Enoxacin, as an antibacterial drug, may be a useful option for cervical cancer treatment [63]. Mitoxantrone is an anti-tumor antibiotic that can be used to treat acute leukemia [64], lymphoma [65], and prostate cancer [66], it also has a certain foundation for the treatment of breast cancer [67] and can also be used to treat multiple sclerosis [68]. Doxorubicin is a recognized therapeutic agent for the treatment of cancers in the blood system. Etoposide is widely used clinically and is used as the standard first-line treatment for a wide range of small cell lung cancer [69]. The above drugs are only based on the drugs related to the genes obtained through the search, and they only provide potential possibilities for the treatment of MPM. To find out whether they can be used in a clinical setting later, more cell experiments and clinical verification are needed.

We used the GEO database to screen the top 10 hub genes related to MPM by using bioinformatics methods. We used GEPIA to verify that the hub genes were all related to the poor prognosis of MPM. Searching for drugs related to hub genes in DGIdb can provide new directions for the treatment of MPM. However, our study had several limitations. First, further studies with larger sample sizes will be needed. Second, we just analyzed the underlying mechanisms by bioinformatics methods, and they remain to be proven by in vitro and in vivo studies.

\section{Conclusion}

In summary, we selected the top 10 hub genes (TOP2A, CDC20, CDK1, UBE2C, CCNB2, NUSAP1, KIF20A, AURKA, CEP55, and ASPM), molecular mechanisms, and potential therapeutic drugs related to MPM by bioinformatics methods. The results of our study are important for providing a reliable theoretical basis for screening, diagnosis, and prognosis, as well as novel therapeutic targets for MPM.

\section{Statement of Ethics}

This study does not involve ethical and moral constraints, and the data in this article are derived from public databases.

\section{Conflict of Interest Statement}

The authors declare that there are no conflicts of interest.

\section{Funding Sources}

This research received no funding support.

\section{Author Contributions}

X.Z. and L.Y. designed and performed the study and wrote the manuscript; they contributed equally to the manuscript. W.C. participated in analyzing the data and revising the manuscript. M.K. reviewed the manuscript. All authors reviewed the final manuscript.

\section{References}

1 Rusch VW, Giroux D, Kennedy C, Ruffini E, Cangir AK, Rice D, et al.; IASLC Staging Committee. Initial analysis of the international association for the study of lung cancer mesothelioma database. J Thorac Oncol. 2012 Nov;7(11):1631-9.

2 Zhou JG, Zhong H, Zhang J, Jin SH, Roudi R, Ma H. Development and Validation of a Prognostic Signature for Malignant Pleural Mesothelioma. Front Oncol. 2019;9:78.

3 Tian K, Bakker E, Hussain M, Guazzelli A, Alhebshi $\mathrm{H}$, Meysami P, et al. p53 modeling as a route to mesothelioma patients stratification and novel therapeutic identification. J Transl Med. 2018 Oct;16(1):282.
4 Feng $\mathrm{H}, \mathrm{Gu} \mathrm{ZY}$, Li Q, Liu QH, Yang XY, Zhang JJ. Identification of significant genes with poor prognosis in ovarian cancer via bioinformatical analysis. J Ovarian Res. 2019 Apr;12(1):35.

$5 \mathrm{Xu}$ Z, Zhou Y, Cao Y, Dinh TL, Wan J, Zhao M. Identification of candidate biomarkers and analysis of prognostic values in ovarian cancer by integrated bioinformatics analysis. Med Oncol. 2016 Nov;33(11):130.

6 Barrett T, Wilhite SE, Ledoux P, Evangelista C, Kim IF, Tomashevsky M, et al. NCBI GEO: archive for functional genomics data sets update. Nucleic Acids Res. 2013 Jan;41(Database issue):D991-5.
7 De Rienzo A, Richards WG, Yeap BY, Coleman MH, Sugarbaker PE, Chirieac LR, et al. Sequential binary gene ratio tests define a novel molecular diagnostic strategy for malignant pleural mesothelioma. Clin Cancer Res. 2013 May;19(9):2493-502.

8 Huang W, Sherman BT, Lempicki RA. Systematic and integrative analysis of large gene lists using DAVID bioinformatics resources. Nat Protoc. 2009;4(1):44-57.

9 Szklarczyk D, Gable AL, Lyon D, Junge A, Wyder S, Huerta-Cepas J, et al. STRING v11: protein-protein association networks with increased coverage, supporting functional discovery in genome-wide experimental datasets. Nucleic Acids Res. 2019 Jan;47(D1):D607-13. 
10 Shannon P, Markiel A, Ozier O, Baliga NS, Wang JT, Ramage D, et al. Cytoscape: a software environment for integrated models of biomolecular interaction networks. Genome Res. 2003 Nov;13(11):2498-504.

11 Chin $\mathrm{CH}$, Chen SH, Wu HH, Ho CW, Ko MT, Lin CY. cytoHubba: identifying hub objects and sub-networks from complex interactome. BMC Syst Biol. 2014;8(Suppl 4):S11.

12 Tang Z, Li C, Kang B, Gao G, Li C, Zhang Z. GEPIA: a web server for cancer and normal gene expression profiling and interactive analyses. Nucleic Acids Res. 2017 Jul; 45(W1):W98-102.

13 Cotto KC, Wagner AH, Feng YY, Kiwala S, Coffman AC, Spies G, et al. DGIdb 3.0: a redesign and expansion of the drug-gene interaction database. Nucleic Acids Res. 2018 Jan; 46(D1):D1068-73.

14 Szklarczyk D, Santos A, von Mering C, Jensen LJ, Bork P, Kuhn M. STITCH 5: augmenting protein-chemical interaction networks with tissue and affinity data. Nucleic Acids Res. 2016 Jan;44(D1):D380-4

15 de Resende MF, Vieira S, Chinen LT, Chiappelli F, da Fonseca FP, Guimarães GC, et al. Prognostication of prostate cancer based on TOP2A protein and gene assessment: TOP2A in prostate cancer. J Transl Med. 2013 Feb;11: 36.

16 Brase JC, Schmidt M, Fischbach T, Sültmann $\mathrm{H}$, Bojar H, Koelbl H, et al. ERBB2 and TOP2A in breast cancer: a comprehensive analysis of gene amplification, RNA levels, and protein expression and their influence on prognosis and prediction. Clin Cancer Res. 2010 Apr;16(8):2391-401.

17 Wang L, Li S, Wang Y, Tang Z, Liu C, Jiao W, et al. Identification of differentially expressed protein-coding genes in lung adenocarcinomas. Exp Ther Med. 2020 Feb;19(2):1103-11.

18 Tang J, Lu M, Cui Q, Zhang D, Kong D, Liao $\mathrm{X}$, et al. Overexpression of ASPM, CDC20, and TTK Confer a Poorer Prognosis in Breast Cancer Identified by Gene Co-expression Network Analysis. Front Oncol. 2019 Apr;9: 310.

19 Yang D, He Y, Wu B, Deng Y, Wang N, Li M, et al. Integrated bioinformatics analysis for the screening of hub genes and therapeutic drugs in ovarian cancer. J Ovarian Res. 2020 Jan;13(1):10.

20 Wu WJ, Hu KS, Wang DS, Zeng ZL, Zhang DS, Chen DL, et al. CDC20 overexpression predicts a poor prognosis for patients with colorectal cancer. J Transl Med. 2013 Jun;11: 142.

21 Palumbo A Jr, Da Costa NM, De Martino M, Sepe R, Pellecchia S, de Sousa VP, et al. UBE2C is overexpressed in ESCC tissues and its abrogation attenuates the malignant phenotype of ESCC cell lines. Oncotarget. 2016 Oct;7(40):65876-87.

22 Gong Y, Wang D, Lin L, Dai J, Yu L. The expression of ubiquitin-conjugating enzyme E2C and KAI1 in ovarian carcinoma and their clinical significance. Medicine (Baltimore). 2019 Nov;98(46):e17896.

23 Zhang Y, Tian S, Li X, Ji Y, Wang Z, Liu C. $\mathrm{UBE} 2 \mathrm{C}$ promotes rectal carcinoma via miR381. Cancer Biol Ther. 2018 Mar;19(3):230-8.
24 Li R, Jiang X, Zhang Y, Wang S, Chen X, Yu $\mathrm{X}$, et al. Cyclin B2 Overexpression in Human Hepatocellular Carcinoma is Associated with Poor Prognosis. Arch Med Res. 2019 Jan; 50(1):10-7.

25 Lei CY, Wang W, Zhu YT, Fang WY, Tan WL. The decrease of cyclin B2 expression inhibits invasion and metastasis of bladder cancer. Urol Oncol. 2016 May;34(5):237.e1-10.

26 Liu Z, Guan C, Lu C, Liu Y, Ni R, Xiao M, et al. High NUSAP1 expression predicts poor prognosis in colon cancer. Pathol Res Pract. 2018 Jul;214(7):968-73.

27 Li L, Lei Q, Zhang S, Kong L, Qin B. Screening and identification of key biomarkers in hepatocellular carcinoma: evidence from bioinformatic analysis. Oncol Rep. 2017 Nov;38(5): 2607-18.

28 Zhang L, Sun L, Zhang B, Chen L. Identification of Differentially Expressed Genes (DEGs) Relevant to Prognosis of Ovarian Cancer by Use of Integrated Bioinformatics Analysis and Validation by Immunohistochemistry Assay. Med Sci Monit. 2019 Dec;25:9902-12.

29 Orenay-Boyacioglu S, Kasap E, Gerceker E, Yuceyar H, Demirci U, Bilgic F, et al. Expression profiles of histone modification genes in gastric cancer progression. Mol Biol Rep. 2018 Dec;45(6):2275-82.

30 Carvalho B, Postma C, Mongera S, Hopmans E, Diskin S, van de Wiel MA, et al. Multiple putative oncogenes at the chromosome $20 \mathrm{q}$ amplicon contribute to colorectal adenoma to carcinoma progression. Gut. 2009 Jan;58(1): 79-89.

31 Caruso S, Calatayud AL, Pilet J, La Bella T, Rekik S, Imbeaud S, et al. Analysis of Liver Cancer Cell Lines Identifies Agents with Likely Efficacy Against Hepatocellular Carcinoma and Markers of Response. Gastroenterology. 2019 Sep;157(3):760-76

32 Lykkesfeldt $\mathrm{AE}$, Iversen $\mathrm{BR}$, Jensen $\mathrm{MB}$, Ejlertsen B, Giobbie-Hurder A, Reiter BE, et al. Aurora kinase A as a possible marker for endocrine resistance in early estrogen receptor positive breast cancer. Acta Oncol. 2018 Jan;57(1):67-73.

33 Schneider MA, Christopoulos P, Muley T, Warth A, Klingmueller U, Thomas M, et al. AURKA, DLGAP5, TPX2, KIF11 and CKAP5: five specific mitosis-associated genes correlate with poor prognosis for non-small cell lung cancer patients. Int J Oncol. 2017 Feb; 50(2):365-72.

34 Lin Y, Fu F, Lv J, Wang M, Li Y, Zhang J, et al. Identification of potential key genes for HER2 positive breast cancer based on bioinformatics analysis. Medicine (Baltimore). 2020 Jan;99(1):e18445.

35 Liu X, Wu J, Zhang D, Bing Z, Tian J, Ni M, Zhang X, et al. Identification of Potential Key Genes Associated with the Pathogenesis and Prognosis of Gastric Cancer Based on Integrated Bioinformatics Analysis. Front Genet. 2018 Jul;9:265.

36 Lin SY, Pan HW, Liu SH, Jeng YM, Hu FC, Peng SY, et al. ASPM is a novel marker for vascular invasion, early recurrence, and poor prognosis of hepatocellular carcinoma. Clin Cancer Res. 2008 Aug;14(15):4814-20.
37 Xie JJ, Zhuo YJ, Zheng Y, Mo RJ, Liu ZZ, Li BW, et al. High expression of ASPM correlates with tumor progression and predicts poor outcome in patients with prostate cancer. Int Urol Nephrol. 2017 May;49(5):817-23.

38 Ayaz P, Andres D, Kwiatkowski DA, Kolbe CC, Lienau P, Siemeister G, et al. Conformational Adaption May Explain the Slow Dissociation Kinetics of Roniciclib (BAY 1000394), a Type I CDK Inhibitor with Kinetic Selectivity for CDK2 and CDK9. ACS Chem Biol. 2016 Jun;11(6):1710-9.

39 Lin SF, Lin JD, Hsueh C, Chou TC, Wong RJ. Potent effects of roniciclib alone and with sorafenib against well-differentiated thyroid cancer. Endocr Relat Cancer. 2018 Oct; 25(10):853-64.

40 Karp JE, Ross DD, Yang W, Tidwell ML, Wei Y, Greer J, et al. Timed sequential therapy of acute leukemia with flavopiridol: in vitro model for a phase I clinical trial. Clin Cancer Res. 2003 Jan;9(1):307-15.

41 Lee DJ, Zeidner JF. Cyclin-dependent kinase (CDK) 9 and 4/6 inhibitors in acute myeloid leukemia (AML): a promising therapeutic approach. Expert Opin Investig Drugs. 2019 Nov;28(11):989-1001.

42 Weiss A, Neubauer MC, Yerabolu D, Kojonazarov B, Schlueter BC, Neubert L, et al. Targeting cyclin-dependent kinases for the treatment of pulmonary arterial hypertension. Nat Commun. 2019 May;10(1):2204.

43 Parry D, Guzi T, Shanahan F, Davis N, Prabhavalkar D, Wiswell D, et al. Dinaciclib (SCH 727965), a novel and potent cyclin-dependent kinase inhibitor. Mol Cancer Ther. 2010 Aug; 9(8):2344-53.

44 Phelps MA, Lin TS, Johnson AJ, Hurh E, Rozewski DM, Farley KL, et al. Clinical response and pharmacokinetics from a phase 1 study of an active dosing schedule of flavopiridol in relapsed chronic lymphocytic leukemia. Blood. 2009 Mar;113(12):2637-45.

45 Cui C, Wang Y, Wang Y, Zhao M, Peng S. Alsterpaullone, a Cyclin-Dependent Kinase Inhibitor, Mediated Toxicity in HeLa Cells through Apoptosis-Inducing Effect. J Anal Methods Chem. 2013;2013:602091.

46 Bae T, Weon KY, Lee JW, Eum KH, Kim S, Choi JW. Restoration of paclitaxel resistance by CDK1 intervention in drug-resistant ovarian cancer. Carcinogenesis. 2015 Dec;36(12): 1561-71.

47 Kang MA, Kim W, Jo HR, Shin YJ, Kim MH, Jeong JH. Anticancer and radiosensitizing effects of the cyclin-dependent kinase inhibitors, AT7519 and SNS-032, on cervical cancer. Int J Oncol. 2018 Aug;53(2):703-12.

48 Dolman ME, Poon E, Ebus ME, den Hartog IJ, van Noesel CJ, Jamin Y, et al. Cyclin-Dependent Kinase Inhibitor AT7519 as a Potential Drug for MYCN-Dependent Neuroblastoma. Clin Cancer Res. 2015 Nov;21(22): 5100-9.

49 Chinn DC, Holland WS, Mack PC. Anticancer activity of the Aurora A kinase inhibitor MK-5108 in non-small-cell lung cancer (NSCLC) in vitro as monotherapy and in combination with chemotherapies. J Cancer Res Clin Oncol. 2014 Jul;140(7):1137-49. 
50 Owonikoko TK, Niu H, Nackaerts K, Csoszi T, Ostoros G, Mark Z, et al.; C14018 study investigators. Randomized Phase II Study of Paclitaxel plus Alisertib versus Paclitaxel plus Placebo as Second-Line Therapy for SCLC: Primary and Correlative Biomarker Analyses. J Thorac Oncol. 2020 Feb;15(2):274-87.

51 Thomas X. Alisertib: a new option for acute myeloid leukaemia. Lancet Haematol. 2020 Feb;7(2):e87-8

52 Binju M, Amaya-Padilla MA, Wan G, Gunosewoyo H, Suryo Rahmanto Y, Yu Y. Therapeutic Inducers of Apoptosis in Ovarian Cancer. Cancers (Basel). 2019 Nov;11(11):1786.

53 Yin T, Zhao ZB, Guo J, Wang T, Yang JB, Wang $C$, et al. Aurora A Inhibition Eliminates Myeloid Cell-Mediated Immunosuppression and Enhances the Efficacy of Anti-PD-L1 Therapy in Breast Cancer. Cancer Res. 2019 Jul;79(13):3431-44.

54 Schöffski P, Besse B, Gauler T, de Jonge MJ Scambia G, Santoro A, et al. Efficacy and safety of biweekly i.v. administrations of the Aurora kinase inhibitor danusertib hydrochloride in independent cohorts of patients with advanced or metastatic breast, ovarian, colorectal, pancreatic, small-cell and nonsmall-cell lung cancer: a multi-tumour, multiinstitutional phase II study. Ann Oncol. 2015 Mar;26(3):598-607.

55 Diamond JR, Eckhardt SG, Pitts TM, van Bokhoven A, Aisner D, Gustafson DL, et al. A phase II clinical trial of the Aurora and angiogenic kinase inhibitor ENMD-2076 for previously treated, advanced, or metastatic triplenegative breast cancer. Breast Cancer Res. 2018 Aug;20(1):82.

56 Diamond JR, Bastos BR, Hansen RJ, Gustafson DL, Eckhardt SG, Kwak EL, et al. Phase I safety, pharmacokinetic, and pharmacodynamic study of ENMD-2076, a novel angiogenic and Aurora kinase inhibitor, in patients with advanced solid tumors. Clin Cancer Res. 2011 Feb;17(4):849-60
57 Borges KS, Andrade AF, Silveira VS, Marco Antonio DS, Vasconcelos EJ, Antonini SR, et al. The aurora kinase inhibitor AMG 900 increases apoptosis and induces chemosensitivity to anticancer drugs in the NCI-H295 adrenocortical carcinoma cell line. Anticancer Drugs. 2017 Jul;28(6):634-44.

58 Arbitrario JP, Belmont BJ, Evanchik MJ, Flanagan WM, Fucini RV, Hansen SK, et al. SNS314 , a pan-Aurora kinase inhibitor, shows potent anti-tumor activity and dosing flexibility in vivo. Cancer Chemother Pharmacol. 2010 Mar;65(4):707-17.

59 Andersen SM, Rosada C, Dagnaes-Hansen F, Laugesen IG, de Darkó E, Dam TN, et al. Topical application of valrubicin has a beneficial effect on developing skin tumors. Carcinogenesis. 2010 Aug;31(8):1483-90.

60 Cookson MS, Chang SS, Lihou C, Li T, Harper SQ, Lang Z, et al. Use of intravesical valrubicin in clinical practice for treatment of nonmuscle-invasive bladder cancer, including carcinoma in situ of the bladder. Ther Adv Urol. 2014 Oct;6(5):181-91.

61 Steenbruggen TG, Steggink LC, Seynaeve CM, van der Hoeven JJM, Hooning MJ, Jager A, et al. High-Dose Chemotherapy With Hematopoietic Stem Cell Transplant in Patients With High-Risk Breast Cancer and 4 or More Involved Axillary Lymph Nodes: 20-Year Follow-up of a Phase 3 Randomized Clinical Trial. JAMA Oncol. 2020 Jan;6(4): 528-34.

62 Li J, Yu K, Pang D, Wang C, Jiang J, Yang S, et al.; CBCSG010 Study Group. Adjuvant Capecitabine With Docetaxel and Cyclophosphamide Plus Epirubicin for TripleNegative Breast Cancer (CBCSG010): An Open-Label, Randomized, Multicenter, Phase III Trial. J Clin Oncol. 2020 Jun;38(16): 1774-84.

63 McDonnell AM, Pyles HM, Diaz-Cruz ES, Barton CE. Enoxacin and Epigallocatechin Gallate (EGCG) Act Synergistically to Inhibit the Growth of Cervical Cancer Cells in Culture. Molecules. 2019 Apr;24(8):1580.

64 Pardee TS, Anderson RG, Pladna KM, Isom S, Ghiraldeli LP, Miller LD, et al. A Phase I Study of CPI-613 in Combination with HighDose Cytarabine and Mitoxantrone for Relapsed or Refractory Acute Myeloid Leukemia. Clin Cancer Res. 2018 May;24(9):206073.

65 Gyan E, Damotte D, Courby S, Sénécal D, Quittet P, Schmidt-Tanguy A, et al.; GOELAMS Group. High response rate and acceptable toxicity of a combination of rituximab, vinorelbine, ifosfamide, mitoxantrone and prednisone for the treatment of diffuse large B-cell lymphoma in first relapse: results of the R-NIMP GOELAMS study. Br J Haematol. 2013 Jul;162(2):240-9.

66 Hakenberg OW, Perez-Gracia JL, Castellano D, Demkow T, Ali T, Caffo O, et al. Randomised phase II study of second-line olaratumab with mitoxantrone/prednisone versus mitoxantrone/prednisone alone in metastatic castration-resistant prostate cancer. Eur J Cancer. 2019 Jan;107:186-95.

67 Baltes F, Pfeifer V, Silbermann K, Caspers J, Wantoch von Rekowski K, Schlesinger M, et al. $\beta 1$-Integrin binding to collagen type 1 transmits breast cancer cells into chemoresistance by activating ABC efflux transporters. Biochim Biophys Acta Mol Cell Res. 2020 May;1867(5):118663.

68 He A, Merkel B, Brown JW, Zhovits Ryerson L, Kister I, Malpas CB, et al.; MSBase study group. Timing of high-efficacy therapy for multiple sclerosis: a retrospective observational cohort study. Lancet Neurol. 2020 Apr; 19(4):307-16.

69 Rudin CM, Ismaila N, Hann CL, Malhotra N, Movsas B, Norris K, et al. Treatment of SmallCell Lung Cancer: American Society of Clinical Oncology Endorsement of the American College of Chest Physicians Guideline. J Clin Oncol. 2015 Dec;33(34):4106-11. 
